\title{
THE STUDY OF THE HYDROGEL SYSTEMS WITH MICELLAR NANODOMAINS AND THE EFFECT OF THE PH CHANGES ON THEIR PROPERTIES
}

\author{
${ }^{1}$ Richard HEGER, ${ }^{1}$ Filip MRAVEC, ${ }^{1}$ Miloslav PEKAř \\ ${ }^{1}$ Brno University of Technology, Faculty of Chemistry, Brno, Czech Republic, EU, xchegerr@fch.vutbr.cz
}

https://doi.org/10.37904/nanocon.2020.3734

\begin{abstract}
Interaction between polyelectrolyte and oppositely charged surfactant leads to a formation of a hydrogel with micellar nanodomains. The main focus of this work was to monitor and study how $\mathrm{pH}$ and its changes affect mainly mechanical properties of phase-separated hydrogels with micellar nanodomains. This work focuses on interaction of hyaluronan and Septonex (carbethoxypendecinium bromide). The studied pH range was 4-9. Rheology was the main technique to study the mechanical properties of the hydrogels. The rheology results were obtained for all prepared hydrogels at the $\mathrm{pH}$ range and as well for hydrogels that had changed $\mathrm{pH}$ after their preparation. The $\mathrm{pH}$ values were always changed to the limit values of the selected range, i.e. four and nine. The results show that the mechanical properties and partly the internal structure of hydrogels vary according to the $\mathrm{pH}$ of their dispersion medium. By changing the $\mathrm{pH}$ to limit values, it was possible to strengthen or soften the bonds within the hydrogel.
\end{abstract}

Keywords: Hydrogel, pH, rheology, hyaluronan, Septonex

\section{INTRODUCTION}

Interaction polyelectrolyte-surfactant is widely researched topic especially for its application potential. The phase separation method makes it possible to prepare physical hydrogels by the interaction of these substances. Hydrogels are known for their ability to absorb a huge amount of water into its structure and thus increase its volume. This fact is used especially in medical applications [1] and for example hydrogels are ideal candidates for use as a drug carrier. This carrier should guarantee a prolonged circulation of the drug and due to its specific structure release the drug slowly and gradually. Its structure also guarantees the passage of oxygen and others nutrients [2].

When working with hydrogels focused on medical applications that could come into contact with the human body, then the conditions that change in this way must be taken into account. One of these conditions is the $\mathrm{pH}$ value, which is a significant factor for all physical interactions. Whether it is the isoelectric point of amino acids, peptides or proteins at which they have total zero charge. It is also the maintenance of a constant $\mathrm{pH}$ that is one of the basic tasks of the internal environment [3].

The application of the hydrogel determines what $\mathrm{pH}$ value the hydrogel will be exposed to. The $\mathrm{pH}$ values of the human body varies considerably. When applied to the skin, the hydrogel may be exposed to $\mathrm{pH}$ in the range of 4 - 7.4 [4]. Upon ingestion, the hydrogel is immediately exposed to the $\mathrm{pH}$ of saliva (6.2 - 7.6) [5], then proceeds up to the stomach where it is exposed to a significantly more acidic $\mathrm{pH}$ (1.7 - 6.7) [6] and then the undigested residue would theoretically be exposed to $\mathrm{pH} 4$ - 7 [7]. In case of vaginal application, it would get in contact with $\mathrm{pH} 3.5$ - 4.3 [8].

The aim of this work was to monitor and describe changes in phase-separated hydrogels caused by a change of the $\mathrm{pH}$ value. The range of studied $\mathrm{pH}$ values correlates with the $\mathrm{pH}$ values that the hydrogel could encounter in the human body. As starting materials for formation of hydrogels by polyelectrolyte-surfactant interactions, a negatively charged polyelectrolyte was selected hyaluronan and the positively charged surfactant Septonex. 
Characterization of the mechanical properties of the prepared hydrogels was performed using rheological methods. All the characteristics were compared between individual gels prepared at different $\mathrm{pH}$ values and as well for hydrogels that had changed $\mathrm{pH}$ after preparation. The $\mathrm{pH}$ values were always changed to the limit values of the selected range, i.e. four and nine.

\section{MATERIALS AND METHODS}

Hydrogels were prepared by mixing the powder substances and then adding the dispersion environment. As a negatively charged polyelectrolyte was used the sodium form of hyaluronan (HyA). In this study the hyaluronan was used at high molecular weight $(1540 \mathrm{kDa})$. As a positively charged surfactant was used carbethopendecinium bromide (Septonex). Both of these substances were mixed together in the clear vial and dissolved in desired buffer. The contents of the vial were for thorough mixing placed on the Vortex shaker and mixed for a brief moment. Thus prepared hydrogel was left to stand for at least 24 hours. The ionic strength of all prepared hydrogels was adjusted to the same value with sodium chloride. Final concentrations of substances were $1 \mathrm{wt} \%$ for HyA and $100 \mathrm{mM}$ for Septonex. Hydrogels were prepared at different $\mathrm{pH}$ values $(4,5,6,7,8$ and 9$)$. The $\mathrm{pH}$ values were adjusted with buffers. Specifically these buffers were citrate-phosphate buffer and borax-hydrochloric acid buffer.

Rheology was the main technique to study the mechanical properties of these hydrogels. The rheology results were obtained for all prepared hydrogels at the $\mathrm{pH}$ range and as well for hydrogels that had changed $\mathrm{pH}$ after their preparation (i.e. 4 and 9). These gels were measured on the AR-G2 rheometer from TA Instruments. The measurement was performed using plate-plate geometry (a diameter of $8 \mathrm{~mm}$, gap size $500 \mu \mathrm{m}$ ). After dosing the sample onto the instrument plate, few drops of silicone oil were added to the sample to prevent it from drying for the duration of the measurement. Before each measurement, a relaxation step was introduced, which allowed the sample to relax for five minutes at the set temperature of $25^{\circ} \mathrm{C}$. For the purposes of this work, strain sweep and frequency sweep tests were performed.

\section{RESULTS AND DISCUSSION}

\subsection{Strain sweep tests}

The first test that was performed was strain sweep test. This test makes possible to detect linear viscoelastic region (LVR). This area tells us where the moduli are independent on the amplitude of deformation. The dependence of the viscous and elastic moduli was determined at a constant frequency value and varying amplitude deformations. With this test we detected the LVR in the range of $0.01-32 \%$. During the whole test the elastic modulus ( $G^{\prime}$ ) prevailed, which indicates that it is a gel. This course and predominance of the elastic modulus were identical for all measured samples. From this range the value of the appropriate amplitude of deformation was chosen. The chosen value was $1 \%$ and this value falls into LVR of all samples. This value was used when setting up the instrument for the oscillation tests for all the samples.

From the value of the end of LVR it is possible to draw conclusions about the mechanical properties of the prepared hydrogels, more specifically the bond strength. These results are summarized in Table 1 . The bigger the value of the end of LVR, the stronger bonds should be and longer resist the compression of the sensor. From the table below it is clear that the strongest gel was a sample prepared at $\mathrm{pH} \mathrm{9.} \mathrm{On} \mathrm{the} \mathrm{contrary,} \mathrm{the}$ softest was the gel prepared at $\mathrm{pH} 4$, which, however, had very close results as other samples. The end of LVR for the other samples occurred at very similar values of amplitude of deformation, from which it can be deduced that these hydrogels are very similar and only the samples prepared at $\mathrm{pH} 9$ differs significantly. Other values that we can find in the Table 1 are the average values of the elastic (G') and viscous (G') moduli in the LVR. The values of these moduli describe the amount of formed gel. Samples with higher moduli values produce more gel and vice versa. Samples with lower values of moduli absorb a larger amount of dispersion 
medium. According to the Table 1, the most of a gel should form in the sample prepared at $\mathrm{pH} 9$ and gel prepared at $\mathrm{pH} 7$ should absorb the most dispersive medium.

Table 1 Data acquired from the strain sweep tests for all the gels prepared at different pH (end of LVR, G', G")

\begin{tabular}{|c|c|c|c|}
\hline $\mathrm{pH}$ & End of LVR (\%) & $G^{\prime}(\mathrm{Pa})$ & $\mathrm{G}^{\prime \prime}(\mathrm{Pa})$ \\
\hline 4 & 9.99 & $2370 \pm 39$ & $925 \pm 45$ \\
\hline 5 & 14.69 & $2951 \pm 48$ & $1205 \pm 30$ \\
\hline 6 & 10.03 & $1871 \pm 18$ & $792 \pm 16$ \\
\hline 7 & 10.01 & $1145 \pm 15$ & $503 \pm 7$ \\
\hline 8 & 14.69 & $3333 \pm 29$ & $1379 \pm 17$ \\
\hline 9 & 31.84 & $4886 \pm 57$ & $701 \pm 22$ \\
\hline
\end{tabular}

In Table 2 we can find the end of LVR values for the samples with changed $\mathrm{pH}$ values. The values of the end of LVR were the highest in the samples that had changed pH to 9. All samples had almost the same LVR end, which means they all should have equally strong bonds. However these bonds should be weaker than the sample prepared at $\mathrm{pH}$ 9. Samples with the $\mathrm{pH}$ value changed to 4 had almost an identical LVR end value as a sample prepared at $\mathrm{pH}$ 4. The strange sample is the sample originally prepared at $\mathrm{pH} 7$. According to the measured values it should have softer bonds than the original sample prepared at $\mathrm{pH} 4$, because its LVR end value is the smallest. It can be explained that the sample, from which this hydrogel was derived before the $\mathrm{pH}$ change, had weaker bonds than other samples, which were based on hydrogels with stronger bonds. Comparison of the above mentioned can be seen in Figure 1.
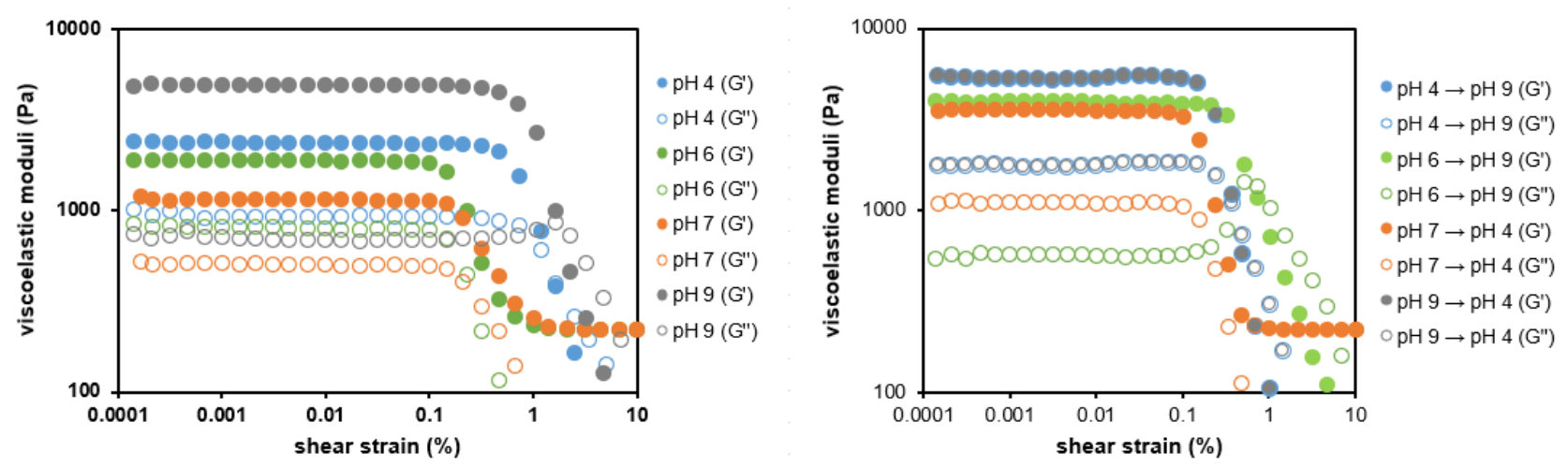

Figure 1 Dependence of viscoelastic moduli on amplitude of deformation for samples prepared at different $\mathrm{pH}$ value (left) and samples with changed $\mathrm{pH}$ environment (right)

Table 2 Data acquired from the strain sweep tests for all the gels witch exchanged pH (end of LVR, G', G")

\begin{tabular}{|c|c|c|c|}
\hline $\mathbf{p H}$ & End of LVR $(\%)$ & $\mathbf{G}^{\prime}(\mathbf{P a})$ & $\mathbf{G} \mathbf{~}^{\prime}(\mathbf{P a})$ \\
\hline $4 \rightarrow 9$ & 14.71 & $3525 \pm 44$ & $511 \pm 21$ \\
\hline $5 \rightarrow 9$ & 14.69 & $4369 \pm 20$ & $700 \pm 12$ \\
\hline $6 \rightarrow 9$ & 14.67 & $3948 \pm 45$ & $570 \pm 12$ \\
\hline $7 \rightarrow 4$ & 6.84 & $3559 \pm 33$ & $1110 \pm 15$ \\
\hline $8 \rightarrow 4$ & 10.02 & $4203 \pm 72$ & $1297 \pm 31$ \\
\hline $9 \rightarrow 4$ & 10.05 & $5320 \pm 80$ & $1778 \pm 35$ \\
\hline
\end{tabular}




\subsection{Frequency sweep tests}

This test is another oscillatory test which measures the dependency of elastic and viscous moduli on the frequency of oscillations. These measurements take place at a constant value of the amplitude deformation and varying frequency of sensor oscillations. In the Figure 2, which is describing the course of dependence of viscoelastic moduli on the increasing frequency of oscillation for samples prepared at different values of $\mathrm{pH}$ and as well samples prepared with changed $\mathrm{pH}$ environment, can be observed gel behaviour. At the beginning of the measurement, the component of the viscous modulus prevailed and increasing the frequency of oscillation led to the point of intersection of the curves, so called cross-over point. Where the values of viscous (G') and elastic (G') moduli are equal. In the beginning, the measurement deforms weak bonds and increasing frequency of oscillation deforms the strong bonds as well. This is indicated in the Figure 2 by the steep rise of elastic modulus. Since the amplitude of deformation from the LVR was chosen for this measurement, it is elastic deformation and after stopping the oscillation, the sample would return to its original state.
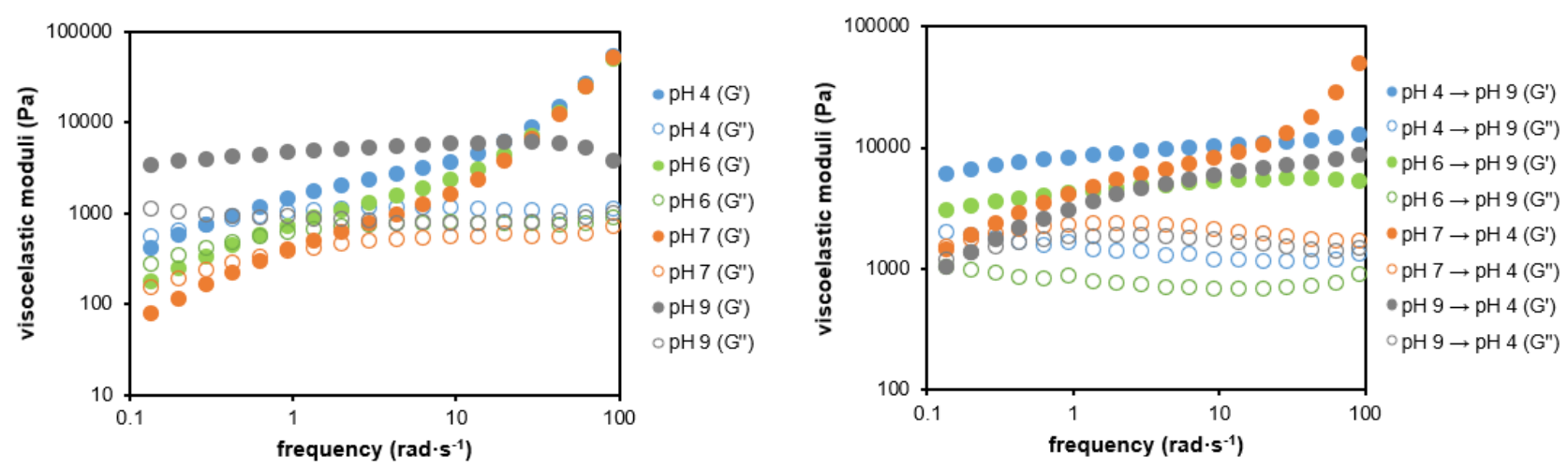

Figure 2 Dependence of viscoelastic moduli on frequency of oscillation for samples prepared at different $\mathrm{pH}$ values (left) and samples with changed $\mathrm{pH}$ environment (right)

Using the Rheology Advantage Data Analysis software, it was possible to record the frequency of oscillation and values of viscoelastic moduli at the cross-over point for all measured samples. These values are summarized in Table 3.

Table 3 Data acquired from frequency sweep test for all gels at different $\mathrm{pH}$ at cross-over point (frequency, viscoelastic moduli)

\begin{tabular}{|c|c|c|}
\hline $\mathbf{p H}$ & Frequency $\left(\mathbf{r a d} \cdot \mathbf{s}^{-1}\right)$ & Viscoelastic moduli (Pa) \\
\hline 4 & 0.296 & 808 \\
\hline 5 & 0.395 & 709 \\
\hline 6 & 0.545 & 581 \\
\hline 7 & 0.843 & 336 \\
\hline 8 & $0.394 \quad$ No cross-over point \\
\hline 9 & \multicolumn{2}{|c|}{} \\
\hline
\end{tabular}

It can be seen from the Table $\mathbf{3}$ that the modulus value, which corresponds with cross-over point that it was highest for the sample prepared at $\mathrm{pH} 4$. The oscillation frequency value for this sample is on the contrary the smallest. This means that the elastic modulus $\left(G^{\prime}\right)$ starts to prevail in the sample sooner than at the other samples. What can be further observed from the table is that the value of the modulus is gradually decreasing with increasing $\mathrm{pH}$ up to the sample prepared at $\mathrm{pH}$ 7. In this sample the lowest value of the module and 
highest value of the oscillation frequency were measured. That indicates that this sample should have the greatest distances between the individual nodes, which stabilize the structure. This is supported by the visual characterisation of this sample itself and the thermogravimetric tests, from which it was found that this sample is able to accept the largest amount of water. On the contrary it was with the sample prepared at $\mathrm{pH} 9$. In this case the moduli G' and G" did not intersect in the measured range of the sample (Figure 2). Thus, in this sample predominates the elastic modulus. Unlike hydrogel prepared at $\mathrm{pH} 7$, this hydrogel should have much smaller distances between individual nodes, which should indicate its impaired permeability. That was again supported by thermogravimetric measurements, from which it turned out that the sample was able to absorb the smallest amount of water.

In Table 4, the cross-over point values for the samples with exchanged $\mathrm{pH}$ values were recorded. Samples prepared at $\mathrm{pH} 4,5$ and 6 had their $\mathrm{pH}$ adjusted to 9 . Samples prepared at $\mathrm{pH} 7,8$ and 9 had their $\mathrm{pH}$ changed to 4 . Immediately it can be noted that the samples, which were changed to $\mathrm{pH} 9$, did not have the cross-over point. This means that samples prepared at $\mathrm{pH} 4,5$ and 6, which had the cross-over point in their initial measured range, had their structure changed simply by changing the $\mathrm{pH}$ value of their environment. The fact that there is no cross-over point means that these samples are very similar to samples already prepared at $\mathrm{pH}$ 9. Conversely, at samples prepared at $\mathrm{pH} 7,8$ and 9 , which had the $\mathrm{pH}$ of their environment changed to 4 , we could observe increase of the viscoelastic moduli in the cross-over point above the values of original samples. The frequency of oscillation were lower than at the sample prepared at $\mathrm{pH} 4$. This indicates that changing the $\mathrm{pH}$ will not convert the sample to a perfect copy of the sample prepared at $\mathrm{pH} 4$, but that the structure retains some of the original bonds.

Table 4 Data acquired from the frequency sweep test for all the gels with exchanged $\mathrm{pH}$ at cross-over point (frequency, viscoelastic moduli)

\begin{tabular}{|c|c|c|}
\hline $\mathbf{p H}$ & Frequency $\left(\mathbf{r a d} \cdot \mathbf{s}^{-1}\right)$ & Viscoelastic moduli (Pa) \\
\hline $4 \rightarrow 9$ & \multicolumn{2}{|c|}{ No cross-over point } \\
\hline $5 \rightarrow 9$ & \multicolumn{2}{|c|}{ No cross-over point } \\
\hline $6 \rightarrow 9$ & \multicolumn{2}{|c|}{ No cross-over point } \\
\hline $7 \rightarrow 4$ & 0.179 & 1444 \\
\hline $8 \rightarrow 4$ & 0.174 & 1045 \\
\hline $9 \rightarrow 4$ & 0.199 & 1199 \\
\hline
\end{tabular}

\section{CONCLUSION}

Twelve hydrogel samples were prepared to study the mechanical properties of hydrogels prepared by phaseseparation. Six samples were prepared at the $\mathrm{pH}$ range 4-9 and the next six samples were prepared at the same $\mathrm{pH}$ values but they had their $\mathrm{pH}$ values of their environment changed before the measurement. In this work was presented detailed comparison of mechanical properties by rheological tests. First of all, the gels were studied by strain sweep tests which demonstrated that the softest hydrogel was prepared at $\mathrm{pH} 4$ with very close values to gel at $\mathrm{pH} 5$ and 6 . The hardest gel was prepared at $\mathrm{pH} 9$. From the results we could draw conclusion about the amount of prepared gel. According to the results the gel formed at $\mathrm{pH} 9$ consisted of the largest amount of gel and conversely the smallest amount of absorbed water. In contrast the gel prepared at $\mathrm{pH} 7$ consisted of the largest amount of absorbed water. Results for the altered $\mathrm{pH}$ values showed that the samples which changed to $\mathrm{pH} 9$ all had the same strong bonds, but these bonds were weaker than the bonds of the sample originally prepared at $\mathrm{pH} 9$. In contrast, the samples which had the $\mathrm{pH}$ changed to 4 all had equally strong bonds as sample prepared at $\mathrm{pH} 4$. 
Complementary results were obtained from frequency sweep tests. After comparing all the values of the crossover points we found that the sample prepared at $\mathrm{pH} 7$ had the greatest distances between individual nodes which stabilize the structure. This predestines it for the best and easiest water absorption. On the contrary for the hydrogel prepared at $\mathrm{pH} 9$, the elastic and viscous moduli did not intersect. In this hydrogel the elastic modulus predominated and the distance between individual nodes is much smaller than for other gels. That's why this hydrogel has very low water permeability. In this direction, the hydrogel prepared at pH 9 appears to be an ideal candidate as $s$ drug carrier with sustained release. For the altered results we recorded that all of cross-over point values were shifted by just the $\mathrm{pH}$ change. However these values were not identical to the values obtained for original hydrogels. This proves that a change in the $\mathrm{pH}$ after preparation can change the mechanical properties of hydrogels but at the same time, we do not create exact copies of original hydrogels. These result can be transferred to custom preparation of hydrogel depending on the application.

\section{REFERENCES}

[1] KIZILAY, E., KAYITMAZER, A.B., DUBIN, P.L. Complexation and coacervation of polyelectrolytes with oppositely charged colloids. Advances in Colloid and Interface Science. 2011, vol. 167, no. 1-2, pp 24-37.

[2] AMIN, S., RAJABNEZHAD, S., KOHLI, K. Hydrogels as potential drug delivery systems. Scientific Research and Essay. 2009, vol. 3, no. 11, pp 1175-1183.

[3] AHMED, E.M. Hydrogel: Preparation, characterization, and applications. Journal of Advanced Research. 2015, vol. 6, no. 2, pp. 105-121.

[4] DARGAVILLE, T.R., FARRUGIA, B.L., BROADBENT, J.A., PACE, S., UPTON, Z., VOELCKER, N.H. Sensors and imaging for wound healing: A review. Biosensors and Bioelectronics. 2013, vol. 41, pp. 30-42.

[5] BALIGA, S., MUGLIKAR, S., KALE, R. Salivary pH: A diagnostic biomarker. Journal of Indian Society of Periodontology. 2013, vol. 17, no. 4.

[6] DRESSMAN, J.B., BERARDI, R.R., DERMENTZOGLOU, L.C., RUSSEL, TL., SCHMALTZ, S.P., BARNETT, J.L., JARVENPAA, K.M. Upper Gastrointestinal (Gl) pH in Young, Healthy Men and Women. Pharmaceutical Research.1990, vol. 7, no. 7, pp. 756-761.

[7] KOZIOLEK, M., GRIMM, M., BECKER, D., IORDANOV, V., ZOU, H., SHIMIZU, J., WANKE, C., GARBACZ, G., WEITSCHIES, W. Investigation of $\mathrm{pH}$ and Temperature Profiles in the GI Tract of Fasted Human Subjects Using the Intellicap $\circledast$ System. Journal of Pharmaceutical Sciences. 2015, vol. 104, no. 9, pp. 2855-2863.

[8] O'HANLON, D.E., COME, R.A., MOENCH, T.R. Vaginal pH measured in vivo: lactobacilli determine $\mathrm{pH}$ and lactic acid concentration. BMC Microbiology. 2019, vol. 19, no. 1. 